\title{
Immunoglobulin replacement treatment by rapid subcutaneous infusion
}

\author{
J Gaspar, B Gerritsen, A Jones
}

\begin{abstract}
Long term intravenous immunoglobulin (IVIG) infusion is an effective treatment for children with immunodeficiencies, but can be complicated by poor venous access, systemic adverse reactions, and the need for frequent hospital admission. Rapid subcutaneous immunoglobulin (SCIG) infusion has been found to be effective in adults with primary immunodeficiency. Twenty six children were treated with SCIG for a median period of two years (range six months to 3.5 years). Fifteen children had previously been treated with IVIG. Retrospective analysis showed that trough IgG concentrations while receiving SCIG were comparable with those while receiving IVIG during maintenance treatment. In severe hypogammaglobulinaemia, however, initial loading with SCIG or IVIG is probably indicated. During the treatment period there was no systemic adverse reaction nor severe reaction requiring admission to hospital. The subjective impression of all families was a significant improvement in the quality of life. This preliminary experience with SCIG in children suggests that it is an effective, convenient, and well tolerated alternative to intravenous treatment. Larger prospective studies are required to determine the place of SCIG in the management of immunodeficiencies. (Arch Dis Child 1998;79:48-51)
\end{abstract}

Keywords: immunoglobulin; subcutaneous infusion; immunodeficiency

Immunoglobulin treatment is the mainstay of treatment for children affected by a variety of immunodeficiency disorders. Inadequate treatment may result in severe life threatening infections and chronic organ damage. ${ }^{1}$

The first description of antibody deficiency was that of Bruton in 1952 and the affected boy was treated with a human immunoglobulin preparation given subcutaneously. ${ }^{2}$ Antibody replacement was subsequently administered intramuscularly until the 1980s, when intravenous immunoglobulin (IVIG) products were widely introduced. Since that time IVIG has become the most popular route of administration. ${ }^{3}$

Many children worldwide have been successfully treated with IVIG, but there are associated disadvantages. Adverse reactions range in severity from mild fever and rash through to rare episodes of severe anaphylaxis. ${ }^{4}$ Transmission of the hepatitis C virus in some IVIG products has been reported and has led to significant morbidity in those affected, ${ }^{56}$ and mortality in a few. A significant group of children, particularly infants, have poor venous access, which results in multiple attempts at venepuncture for each infusion. In a number of patients indwelling central venous devices have been used, but these are associated with risks of infection and thromboembolic complications. ${ }^{7}$ Although home IVIG treatment programmes have been set up, ${ }^{8}$ most intravenous infusions in small children require inpatient administration, necessitating regular visits to hospital with a resulting loss of nursery, school, and family time, and considerable commitment from the family. The cost of regular IVIG is high, both because of the cost of the products and the associated costs of hospital based care.

Improved recognition of antibody deficiency is resulting in the increasing use of immunoglobulin replacement treatment. The treatment of very young children highlights the need to find a safe, convenient, and effective alternative to intravenous administration. There are reports of slow subcutaneous infusions of immunoglobulin (1-3 $\mathrm{ml} /$ hour) in both adults and children. ${ }^{910}$ These were not popular, however, because infusions were given over 12 hours, and they were never widely introduced. More recently, rapid infusions $(20+\mathrm{ml} /$ hour $)$ have been favourably reported in adults ${ }^{11}$ and in a small number of children. ${ }^{12}{ }^{13}$ We report our early experience with 26 children in whom replacement immunoglobulin treatment was given by the rapid subcutaneous method.

\section{Methods}

PATIENTS

Twenty six children (17 boys and nine girls aged 1.5 months to 15 years) were treated for periods of six months to 3.5 years. Eleven were treated with subcutaneous immunoglobulin (SCIG) from the outset (group A) and 15 patients had previously received IVIG (group B). The reasons for the changes to subcutaneous treatment were poor venous access in 12 patients and personal request in three.

Immunological diagnoses in these children were severe combined immunodeficiency (one child), combined immunodeficiency (three children), $\mathrm{X}$ linked agammaglobulinaemia (three children), panhypogammaglobulinaemia (seven children), IgG subclass deficiency (four children) with associated IgA or IgM deficiency, or both (five children), or associated enteropathy (three children). 
Table 1 Evaluation of parental satisfaction (abstract of questionnaire) - group $A(n=9)$

\begin{tabular}{lll}
\hline & Agree & Disagree \\
\hline We fit the infusions conveniently into our lifestyle & 9 & 0 \\
The subcutaneous injections do not cause significant discomfort to my child & 7 & 2 \\
The infusions are easy to prepare & 9 & 0 \\
\hline
\end{tabular}

Table 2 Evaluation of parental satisfaction (abstract of questionnaire)—group B $(n=11)$

\begin{tabular}{lll}
\hline & Less & No change \\
\hline Overall inconvenience compared to IVIG & 9 & 2 \\
Discomfort to your child & 9 & 2 \\
Parents out of pocket expenses & 7 & 4 \\
Adverse after effects of treatment & 4 & 7 \\
Time missed from nursery or school to give infusions & 7 & 4 \\
Time missed from parents' work to give infusions $(\mathrm{n}=7)^{\star}$ & 5 & 2 \\
\hline
\end{tabular}

$\star$ Six families did not complete the questionnaire.

\section{SUBCUTANEOUS INFUSIONS}

All children received a mercury free, $16 \%$ normal human immunoglobulin licensed for intramuscular use (Gammabulin; Immuno Ltd, Newbury, Berks, UK) using a portable infusion pump (Syringe Driver, MS16A; Graseby Medical, Watford, UK). The mean dosage was $160 \mathrm{mg} / \mathrm{kg} /$ week (range 70-260). The lower dosages were used in children with mild immunodeficiency and higher doses in those with severe panhypogammaglobulinaemia. After estimating the desired weekly dose, convenient infusion regimens were set individually. All but one patient received two infusions simultaneously, twice each week in five patients, once a week in 13 patients, and once every 10th day in seven patients. One older patient preferred to have a single infusion five times each week. Infusions were delayed in the event of fever or infection.

Infusions were usually given into subcutaneous tissue in the thigh. The buttocks and abdomen were used successfully in two children, but were unpopular with the rest of the group. Local vasoconstricting anaesthetic cream containing lignocaine and prilocaine (EMLA) was used by 21 patients to ease the discomfort of siting the needles. A fine $25 \mathrm{G}$ butterfly needle attached to a $10 \mathrm{ml}$ syringe was inserted into the subcutaneous tissue at a $45^{\circ}$ angle and taped down with clear adhesive dressing.

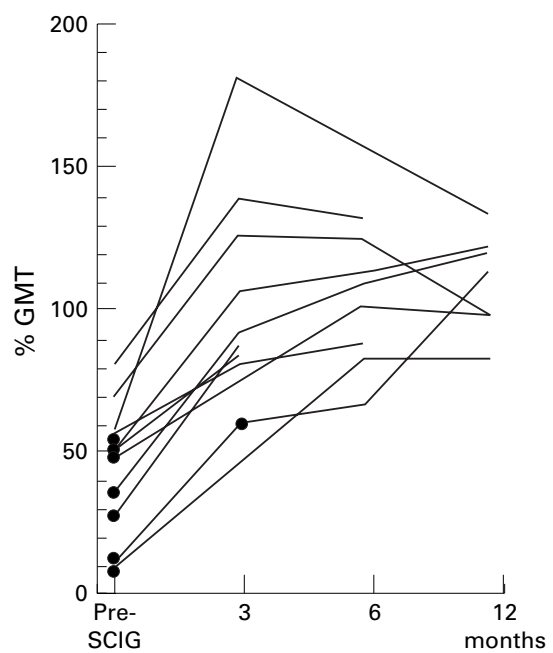

Eight patients with sufficient subcutaneous tissue had $10 \mathrm{ml}$ injected at each site; 10 children could tolerate $7.5 \mathrm{ml}$; and eight children with poorer subcutaneous tissue received $5 \mathrm{ml}$ at each site. We did not attempt to inject any more than $10 \mathrm{ml}$ at each site. No more than two simultaneous infusions per patient were given because of a limited supply of syringe pumps. The first infusion was given at $10 \mathrm{ml} /$ hour and most subsequent infusions at $20 \mathrm{ml} /$ hour.

Twenty four patients were established on home treatment, one preferred to attend hospital, and the remaining patient received infusions at their general practitioner's surgery. Families and, where necessary, community paediatric nurses underwent training in setting up and administering the infusions, the recognition of adverse reactions, and the use of an adrenaline autoinjector pen. All patients were asked to report infectious episodes on their monthly infusion records and were followed up by liberal telephone contact and outpatient appointments at the immunology centre every three to six months.

\section{ANTIBODY MEASUREMENTS AND LIVER FUNCTION} TESTS

Serum immunoglobulin levels were measured by nephelometry before IVIG, before SCIG, and at three, six, and 12 months after starting treatment with SCIG. If values were not available at these time points, the value nearest to that time point was used. IgG concentrations were expressed as a percentage of the geometric mean titre (GMT) of normal age matched controls. $^{1415}$

Blood samples were taken every one to three months for alanine aminotransferase, aspartate aminotransferase, or $\gamma$ glutamyl transferase measurements.

ESTIMATION OF PATIENT SATISFACTION

All families were approached to complete a concise questionnaire to evaluate patient satisfaction; 20 families responded. In group A, parents were asked to respond to certain

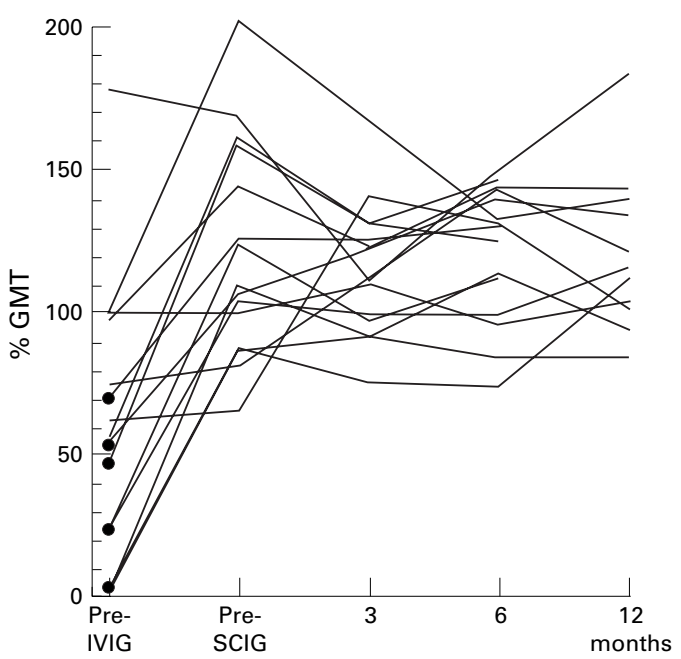

Figure 1 Trough IgG concentrations expressed as a percentage of the geometric mean titre (GMT) of normal age matched controls. Eleven patients received SCIG from the outset (group A); and 15 patients received IVIG followed by SCIG (group B). 
statements (table 1), and in group B, parents were asked to compare SCIG with IVIG after at least six months of receiving SCIG (table 2).

\section{Results}

SERUM IgG CONCENTRATIONS

In group A (SCIG alone, 11 patients), eight patients had a pretreatment GMT for IgG below the normal range of age matched reference values. All patients achieved normal IgG concentrations by three months, except for two children with pretreatment IgG concentrations of less than $20 \%$ of the GMT. All patients achieved normal concentrations at six months (fig 1A).

In group B (previous IVIG, 15 patients), eight patients had a GMT for IgG below the normal range at the initiation of IVIG. All patients had a normal IgG GMT at the time of starting SCIG and these were maintained thereafter (fig 1B).

FREQUENCY OF INFECTION

During the period of observation there was no serious life threatening infections and no infections requiring admission to hospital. Patient records for antibiotic treatment and infection frequency showed, albeit subjectively, that the antibiotic requirement and infection frequency were reduced after starting SCIG in group A and showed no noticeable difference in group B (data not shown).

ADVERSE REACTIONS AND SAFETY

All children developed painless raised lumps over the infusion site, which lasted between two and 24 hours. Ten patients also had local erythema for which no treatment was required. Two patients found the infusions uncomfortable at the faster rate and preferred to run the infusions at $10 \mathrm{ml} /$ hour. Alanine aminotransferase, aspartate aminotransferase, or $\gamma$ glutamyl transferase remained within the normal range in all patients.

\section{PATIENT SATISFACTION}

In general, parents in the two groups responded positively to the SCIG infusions (tables 1 and 2). Additional comments included the ease of setting up home treatment and the significant mobility afforded to children using the portable syringe drivers. Five children, the youngest of whom was aged six years, learnt to place their own needles, which helped in achieving independence from their parents. Nine parents commented that they preferred the shorter infusion time even though the infusions were required more frequently than IVIG. Eleven families, however, identified the higher infusion frequency as the main disadvantage of SCIG. All families said that overall they preferred SCIG treatment to IVIG.

\section{Discussion}

There is a clearly defined need for an alternative to IVIG in a significant proportion of immunodeficient children. The subcutaneous route offers such an alternative; however, this method demands more frequent infusions, given in multiple sites, and there was early doubt about the likely acceptability in view of this.
All human immunoglobulin products carry a small risk of virus transmission. ${ }^{16}$ Outbreaks of hepatitis $C$ have been associated with several intravenous products, but none has been reported with intramuscular products. It is now mandatory for a specific virus inactivation step to be incorporated into the manufacturing process for intravenous products. This requirement has not yet been applied to intramuscular preparations, although hepatitis $\mathrm{C}$ polymerase chain reaction testing becomes mandatory in 1998. It is, however, likely and desirable that the requirement for a specific virus inactivation step for intramuscular products will change in the near future. In our population liver enzymes remained normal throughout treatment.

Initially, SCIG was given only to small children who had poor venous access, but the patient group was gradually expanded as the method proved popular. Two teenage boys with $\mathrm{X}$ linked agammaglobulinaemia requested a change from IVIG because of the increased flexibility and independence of self infusion allowed by SCIG. Audit of the patient group confirmed that many families prefer shorter, more frequent infusions at home to the more disruptive and lengthy three weekly visits to hospital demanded by IVIG.

Maintenance of adequate serum IgG concentrations in adults treated with SCIG has been well documented. ${ }^{17}$ Our observations in children are consistent with this. The doses required are variable, as with the intravenous route. Only one of our patients was loaded rapidly by giving a series of SCIG infusions. In patients with very low IgG, subnormal concentrations after three months may not be acceptable, and loading doses of SCIG or IVIG may be of benefit to achieve normal concentrations more rapidly.

No formal cost comparison with home IVIG has been undertaken, but at present the subcutaneous products are considerably cheaper than the cheapest intravenous products. Home treatment preparation and training can be completed quickly after three to four infusion sessions, and the disposable equipment required is minimal. Our impression is that a formal cost audit of SCIG would show considerable cost savings for the service. Home treatment is not possible for many of the smaller children receiving IVIG, but it is a viable proposition for almost all children receiving SCIG.

Subcutaneous immunoglobulin infusion treatment has, in our experience, been a convenient and popular alternative to intravenous treatment. SCIG is well tolerated and no systemic nor clinically significant adverse effect occurred. These early observations suggest that it is effective and safe, but more formal studies are required in children to verify these impressions, and pharmaceutical companies should be encouraged to develop new generation immunoglobulin products suitable for subcutaneous use to minimise viral infection risk. 
1 Roifman CM, Levison H, Gelfand EW. High dose versus low-dose intravenous immunoglobulin in hypogamma-
globulinaemia and chronic lung disease. Lancet globulinaemia

2 Bruton OC. Agammaglobulinaemias. Pediatrics 1952;9: $722-7$.

3 Primary immunodeficiency diseases: report of a WHO scientific group. Clin Exp Immunol 1997;109:S1-28.

4 Misbah SA, Chapel HM. Adverse effects of intravenous immunoglobulin. Drug Safety 1993;9:254-62.

5 Widell A, Zhang YY, Andersson-Gäre B, Hammerström L. At least three hepatitis C virus strains implicated in Swedish and Danish patients with intravenous immunoglobulin associated hepatitis C. Transfusion 1997;37: 313-20.

6 Echevarria JM, Leon P, Domingo CJ, et al. Laboratory diagnosis and molecular epidemiology of an outbreak of hepatitis $\mathrm{C}$ virus among recipients of human intravenous immunoglobulin in Spain. Transfusion 1996; 36:725-30.

7 Dollery CM, Sullivan ID, Bauraind O, Bull K, Milla P. Pulmonary embolism and long term central venous access for parenteral nutrition. Lancet 1994;344:1043-5.

8 Brennan VM. Home self infusion of IV immunoglobulin. Nursing Standard 1991;5:37-9.

9 Roord JJ, Van de Meer JWM, Kuis W, et al. Home treatment in patients with antibody deficiency by slow subcutaneous infusion of gammaglobulin. Lancet 1982;i:689-90.
10 Ugazio AG, Duse M, Plebani A, Notarangelo LD, Burgio GR. Subcutaneous infusions of gamma globulins in the management of agammaglobulinaemic patients. Birth Defects 1983;19:213-15.

11 Gardulf A, Hammerstrom L, Smith CIE. Home treatment of hypogammaglobulinaemia with subcutaneous gammaglobulin by rapid infusion. Lancet 1991;338:162-6.

12 Thomas MJ, Brennan VM, Chapel HH. Rapid subcutaneous immunoglobulin infusions in children. Lancet 1993; 342:1432-3.

13 Abrahamsen TG, Sanderson H, Bustnes A. Home therapy with subcutaneous immunoglobulin infusions in children
with congenital immunodeficiencies. Paediatrics 1996;98: 1127-31

14 Cejka J, Mood DW, Kim CS. Immunoglobulin concentration in sera of normal children: quantitation against an international reference preparation. Clin Chem 1974;20:656-9.

15 Gerritsen EJA, Tol HJD van, Lankester AC, et al. Immunoglobulin levels and monoclonal gammopathies in children after bone marrow transplantation. Blood 1993;82: 3493-502.

16 Yap PL, McOmish F, Webster ADB, et al. Hepatitis C virus transmission by intravenous immunoglobulin. $\mathcal{F}$ Hepatol 1994;21:455-60.

17 Waniewski J, Gardulf A, Hammarström L. Bioavailability of gamma-globulin after subcutaneous infusions in patients with common variable immunodeficiency. Clin Immunol 1994;14:90-7. 